\title{
Probing the cholinergic system to understand neurodegenerative diseases
}

\author{
"...the discovery of new radioligands is a real challenge for chemists \\ and radiochemists."
}

First draft submitted: 14 November 2016; Accepted for publication: 16 November 2016; Published online: 18 January 2017

Keywords: acetylcholine $\bullet$ acetylcholinesterase $\bullet$ muscarinic acetylcholine receptor - neurodegenerative diseases $\bullet$ nicotinic acetylcholine receptors

The cholinergic system plays an important role in number of cognitive functions, including memory, selective attention and emotional processing [1]. Acetylcholine $(\mathrm{ACh})$ is a ubiquitous neurotransmitter with widespread innervation in the cortex, subcortical structures and the cerebellum. The three main projection systems of the CNS are the nucleus basalis of Meynert, the pedunculopontine nucleus and cholinergic neurons in the striatum. ACh is produced from choline and acetate, as starting substrates, by action of the choline acetyltransferase and produced $\mathrm{ACh}$ is stored into synaptic vesicles by vesicular ACh transporter (VAChT). The two important classes of the postsynaptic cholinergic receptors are the nicotinic receptors (nAChRs) and the muscarinic receptors (mAChRs). The nAChRs are ligand-gated ion channels permeable to cations such as $\mathrm{Na}$, $\mathrm{K}$ and $\mathrm{Ca}$. Neuronal nAChRs are homopentameric and heteropentameric combinations of $\alpha$ and $\beta$ subunits. Among the nAChRs subtypes in the CNS, $\alpha 4 \beta 2$ and $\alpha 7 \mathrm{nAChRs}$ are predominant in the brain and play a role in the neurodegenerative processes [2]. Muscarinic receptors belong to the family of G-protein-coupled receptors (GPCRs) and there are five subtypes of receptors, named M1-M5. M1, M3 and M5 receptors are coupled with Gq proteins, whereas M2 and M4 receptors are coupled with $\mathrm{G}_{\mathrm{i} / \mathrm{o}}$ proteins. M1 receptors are abundantly expressed in brain including the cerebral cortex, hippo- campus and striatum, and they are consequently involved in the learning and memory processes [3].

Altered levels of ACh or modified receptors expression and function in the nervous system have been described in several neurodegenerative diseases such as Alzheimer's disease (AD) and Parkinson's disease - especially with dementia (PDD) $[4,5]$. AD is the most common age-related form of dementia and is characterized by progressive memory loss, decline in language skills, behavioral disturbances and other cognitive impairments. In 2015, WHO estimated 47.5 million people affected by AD worldwide and the prevalence of dementia is expected to reach 135.5 million in 2050. AD has been described as a complex and multifactorial disease and histologically characterized by extra neuronal amyloid- $\beta$ protein deposits and intraneuronal hyperphosphorylated T-protein aggregation. Other cellular hallmarks include oxidative stress, dyshomeostasis of biometals, a loss of synapses and cell death, especially in the cholinergic neurons network [6]. The cholinergic hypothesis submits that the loss of cognitive and memory associated with $\mathrm{AD}$ are based on the observation of a deficiency of ACh in the CNS [7]. Therefore, the most widely used concept for AD drug development during these last 20 years, is especially based on increasing the brain $\mathrm{ACh}$ levels by inhibiting acetylcholinesterase (AChE), the enzyme that hydrolyses ACh in choline and acetate. Although their efficacies are moder-

\author{
Pierre-Yves Renard ${ }^{1}$ \\ \& Ludovic Jean*,1 \\ 'Normandie Univ, INSA Rouen, \\ UNIROUEN, CNRS, COBRA (UMR 6014), \\ 76000, Rouen, France \\ *Author for correspondence: \\ ludovic.jean@univ-rouen.fr
}


ate, $\mathrm{AChE}$ inhibitors (tacrine, galantamine, rivastigmine and donepezil) are the sole approved drugs as palliative treatments in order to restore the cognitive functions of $\mathrm{AD}$ patients. Besides, M1 agonists and positive allosteric modulator (PAM), as well as, $\alpha 7$ agonists and PAMs are also developed as therapeutic approaches to increase cholinergic function in neurodegenerative processes.

In order to understand the role of each receptor and enzyme involved in the cholinergic system and in the neurodegenerative processes, molecular imaging techniques such as positron emission tomography (PET) and single-photon emission computed tomography (SPECT) using specific radioligands have been applied for in vivo imaging, while applications of the fluorescence imaging have been developed for in vitro and in cellulo studies. The latter, cheaper and safer than PET/SPECT imaging system, is a good alternative to localize the receptors as well as to lead kinetic and thermodynamic studies on live or fixed tissues.

\section{"...fluorescence resonance energy transfer assays have been reported to probe human M1 receptors} using fluorescent positive allosteric modulator."

Many efforts have been made to develop radioligands for imaging human brain nAChRs [8]. Radioligands including $\left[{ }^{11} \mathrm{C}\right]$-nicotine, $2-\left[{ }^{18} \mathrm{~F}\right]$-FA and $\left[{ }^{18} \mathrm{~F}\right]$-AZAN have been used in clinical studies for in vivo imaging of $\alpha 4 \beta 2 \mathrm{nAChRs}$ within the brain of $\mathrm{AD}$ and PDD patients [9]. $\left[{ }^{11} \mathrm{C}\right]$-nicotine presents a nonspecific binding of $\alpha 4 \beta 2 \mathrm{nAChRs}$, whereas 2-[18 $\mathrm{F}]-\mathrm{FA}$ or $\left[{ }^{18} \mathrm{~F}\right]-\mathrm{AZAN}$, developed by Hordi et al., are more suitable radioligands for imaging the brain distribution of $\alpha 4 \beta 2 \mathrm{nAChRs}$ in human. Although numerous $\alpha 7 \mathrm{nAChRs}$ agonists have been developed to treat $\mathrm{AD}$, schizophrenia and depression [10], the development of efficient $\alpha 7$ $\mathrm{nAChRs}$ radioligands is more challenging and, nowadays, none of the developed PET or SPECT radiotracers for imaging human brain $\alpha 7 \mathrm{nAChRs}$ shows an efficient binding selectivity for a clinical development. Regarding the fluorescent probes, there are only two fluorescent dyes for imaging nAChRs. Fluorescent epibatidine agonists were ideally suited for $\mathrm{nAChRs}$ in vitro, in cellulo or even native tissues [11], and, more recently, 3-amino-quinuclidine derivatives have been reported as partial agonists of $\alpha 7$ nAChRs. Besides, fluorescent ACh derivatives have also been examined and showed a subnanomolar affinity toward Torpedo nAChRs [12].

Numerous PET/SPECT radioligands for imaging mAChRs, including $\left[{ }^{11} \mathrm{C}\right]-\mathrm{NMPB}$ or $\left[{ }^{123} \mathrm{I}\right]-\mathrm{QNB}$, have been reported for $\mathrm{AD}$ and $\mathrm{PDD}$ patients, nevertheless all these radiotracers showed a low selectivity toward
$\mathrm{mAChRs}$ [13]. Regarding fluorescent imaging of these GPCRs, fluorescence resonance energy transfer assays have been reported to probe human M1 receptors using fluorescent PAM [14].

$\mathrm{AChE}$ is known for decades as a reliable marker for brain cholinergic nerve termini, and the most commonly used radiotracers to image the distribution of AChE in brain are two radiolabeled lipophilic $\mathrm{ACh}$ analogs, $\left[{ }^{11} \mathrm{C}\right]-\mathrm{PMP}$ and $\left[{ }^{11} \mathrm{C}\right]-\mathrm{MP} 4 \mathrm{~A}$, that diffuse readily into brain [15]. Radiolabeling of AChE inhibitors have also been undertaken for mapping cerebral AChE activity. While distribution of $\left[{ }^{11} \mathrm{C}\right]-$ methyl-tacrine and $\left[{ }^{11} \mathrm{C}\right]$-donepezil did not correlate with brain AChE distribution due to a nonspecific binding, (-)-[ $\left[{ }^{11} \mathrm{C}\right]$-galantamine showed an accumulation in the AChE-abundant regions of brain. Nevertheless, none of these radiolabeled AChE ligands are used for imaging human brain AChE in clinical studies [16]. Besides, we have prepared three $\left[{ }^{18} \mathrm{~F}\right]$-huprines known as potent and selective $\mathrm{AChE}$ inhibitors. The radiotracers showed binding in the rat striatum, rich in AChE but low binding in the surrounding cortex, poor in $\mathrm{AChE}$ [17]. Regarding the fluorescent imaging of $\mathrm{AChE}$, we have recently developed two near infrared fluorescent probes HupNIR1 and HupNIR2 based on the huprine scaffold and cyanine 5.0 dye. These probes are the first ones described in the literature showing a great selectivity and sensitivity for active $\mathrm{AChE}$ even in the brain region displaying a low $\mathrm{AChE}$ concentration as striatum [18].

The vesicular cholinergic transporters (VAChT) are localized in the membrane of the presynaptic vesicle where ACh is accumulated, and play an important role in the regulation of cholinergic activity [19]. Many PET/SPECT radiotracers targeting VAChT have been developed, nevertheless [ $\left.{ }^{123} \mathrm{I}\right]-\mathrm{IBVM}$, an analog of vesamicol, is the unique radiotracer used for human in vivo imaging [20]. Regarding the fluorescent VAChT imaging, experiments have been investigated using green fluorescent protein-tagged VAChT in living cholinergic cells [21].

Many PET or SPECT radioligands have been developed to understand the role of receptors or enzyme involved in the dysfunction of cholinergic system observed in neurodegenerative diseases such as AD and PDD. Numerous existing radiotracers show a nonspecificity or a low affinity toward the target, and are unusable for in vivo imaging. Therefore, the discovery of new radioligands is a real challenge for chemists and radiochemists. Besides, the development of fluorescent selective probes for receptors involved in the dysfunction of cholinergic system is also required by biologists and neurobiologists. 


\section{Financial \& competing interests disclosure}

The authors have no relevant affiliations or financial involvement with any organization or entity with a financial interest in or financial conflict with the subject matter or materials discussed in the manuscript. This includes employ-

\section{References}

1 Jackson CE. Cholinergic System. In: Encyclopedia of Clinical Neuropsychology. Kreutzer JS, DeLuca J, Caplan B (Eds). Springer New York, NY, USA. 562-564 (2011).

2 Gotti C, Zoli M, Clementi F. Brain nicotinic acetylcholine receptors: native subtypes and their relevance. Trends Pharmacol. Sci. 27(9), 482-491 (2006).

3 Scarr E. Muscarinic receptors: their roles in disorders of the central nervous system and potential as therapeutic targets. CNS Neurosci. Ther. 18(5), 369-379 (2012).

4 Tata AM, Velluto L, D’Angelo C, Reale M. Cholinergic system dysfunction and neurodegenerative diseases: cause or effect? CNS Neurol. Disord. Drug Targets. 13(7), 1294-1303 (2014).

5 Muller ML, Bohnen NI. Cholinergic dysfunction in Parkinson's disease. Curr. Neurol. Neurosci. Rep. 13(9), 377 (2013).

6 Querfurth HW, LaFerla FM. Alzheimer's disease. N. Engl. J. Med. 362(4), 329-344 (2010).

7 Bartus RT, Dean RL 3rd, Beer B, Lippa AS. The cholinergic hypothesis of geriatric memory dysfunction. Science 217(4558), 408-414 (1982).

8 Wu J, Ishikawa M, Zhang J, Hashimoto K. Brain imaging of nicotinic receptors in Alzheimer's disease. Int. J. Alzheimers Dis. 2010, 548913 (2010).

9 Horti AG, Kuwabara H, Holt DP, Dannals RF, Wong DF. Recent PET radioligands with optimal brain kinetics for imaging nicotinic acetylcholine receptors. J. Labelled Comp. Radiopharm. 56(3-4), 159-166 (2013).

10 Bertrand D, Lee CH, Flood D, Marger F, Donnelly-Roberts D. Therapeutic potential of $\alpha 7$ nicotinic acetylcholine receptors. Pharmacol. Rev. 67(4), 1025-1073 (2015).

11 Grandl J, Sakr E, Kotzyba-Hibert F et al. Fluorescent epibatidine agonists for neuronal and muscle-type nicotinic acetylcholine receptors. Angew. Chem. Int. Ed. Engl. 46(19), 3505-3508 (2007). ment, consultancies, honoraria, stock ownership or options, expert testimony, grants or patents received or pending, or royalties.

No writing assistance was utilized in the production of this manuscript.

12 Krieger F, Mourot A, Araoz R et al. Fluorescent agonists for the Torpedo nicotinic acetylcholine receptor. Chembiochem 9(7), 1146-1153 (2008).

13 Eckelman WC. Imaging of muscarinic receptors in the central nervous system. Curr. Pharm. Des. 12(30), 3901-3913 (2006).

14 Daval SB, Valant C, Bonnet D et al. Fluorescent derivatives of AC-42 to probe bitopic orthosteric/allosteric binding mechanisms on muscarinic M1 receptors. J. Med. Chem. 55(5), 2125-2143 (2012).

15 Kikuchi T, Okamura T, Fukushi K et al. Cerebral acetylcholinesterase imaging: development of the radioprobes. Curr. Top. Med. Chem. 7(18), 1790-1799 (2007).

16 Kimura H, Kawai T, Hamashima Y et al. Synthesis and evaluation of $(-)$ - and $(+)-\left[{ }^{11} \mathrm{C}\right]$ galanthamine as PET tracers for cerebral acetylcholinesterase imaging. Bioorg. Med. Chem. 22(1), 285-291 (2014).

17 Da Costa Branquinho E, Becker G, Bouteiller C, Jean L, Renard PY, Zimmer L. Radiosynthesis and in vivo evaluation of fluorinated huprine derivates as PET radiotracers of acetylcholinesterase. Nucl. Med. Biol. 40 (4), 554-560 (2013).

18 Chao S, Krejci E, Bernard V, Leroy J, Jean L, Renard PY. A selective and sensitive near-infrared fluorescent probe for acetylcholinesterase imaging. Chem. Commun. 52(77), 11599-11602 (2016).

19 Prado VF, Roy A, Kolisnyk B, Gros R, Prado MA. Regulation of cholinergic activity by the vesicular acetylcholine transporter. Biochem. J. 450 (2), 265-274 (2013).

20 Kuhl DE, Koeppe RA, Fessler JA et al. In vivo mapping of cholinergic neurons in the human brain using SPECT and IBVM. J. Nucl. Med. 35(3), 405-410 (1994).

21 Barbosa J Jr, Ferreira LT, Martins-Silva C et al. Trafficking of the vesicular acetylcholine transporter in SN56 cells: a dynamin-sensitive step and interaction with the AP-2 adaptor complex. J. Neurochem. 82(5), 1221-1228 (2002). 